\title{
Designing irrigation pipes
}

\author{
Antonio Fasano • Angiolo Farina
}

Received: 18 April 2011 / Accepted: 18 August 2011 / Published online: 18 August 2011

(C) 2011 Fasano, Farina; licensee Springer. This is an Open Access article distributed under the terms of the Creative Commons Attribution License

\begin{abstract}
The use of porous ducts to deliver water to agricultural fields is an old technique which helps saving water and prevents ground erosion. Designing porous duct is not as a simple task as it looks and apparently has never been the subject of mathematical research. Here the problem is addressed making use of a double rescaling of space and velocity variables, which allows the derivation of the governing equations starting from the study of the classical Navier Stokes equations in a pipe. Such equations are then solved obtaining results of practical interest in design of irrigation pipes, both for low discharge pipes (small plants) and for high discharge pipes (large plants).
\end{abstract}

Keywords Porous pipes · Navier Stokes equations · asymptotic expansions · irrigation

Mathematics Subject Classification 76S05 - 76D05 - 35C20

\section{List of principal symbols}

$A, B, C$ constants (33), (34), (26)

$h \quad$ water level in reservoir

$H \quad$ pipe outer radius

$K^{*} \quad$ pipe wall permeability

$N$ number of dripping tubes or slots

$L^{*} \quad$ pipe length

$S^{*} \quad$ wall thickness

A. Fasano $(\bowtie) \cdot$ A. Farina

Dipartimento di Matematica "Ulisse Dini”, Università degli Studi di Firenze, Viale Morgagni 67/A, I-50134 Firenze, Italy

e-mail: fasano@math.unifi.it

A. Farina

e-mail: farina@math.unifi.it 


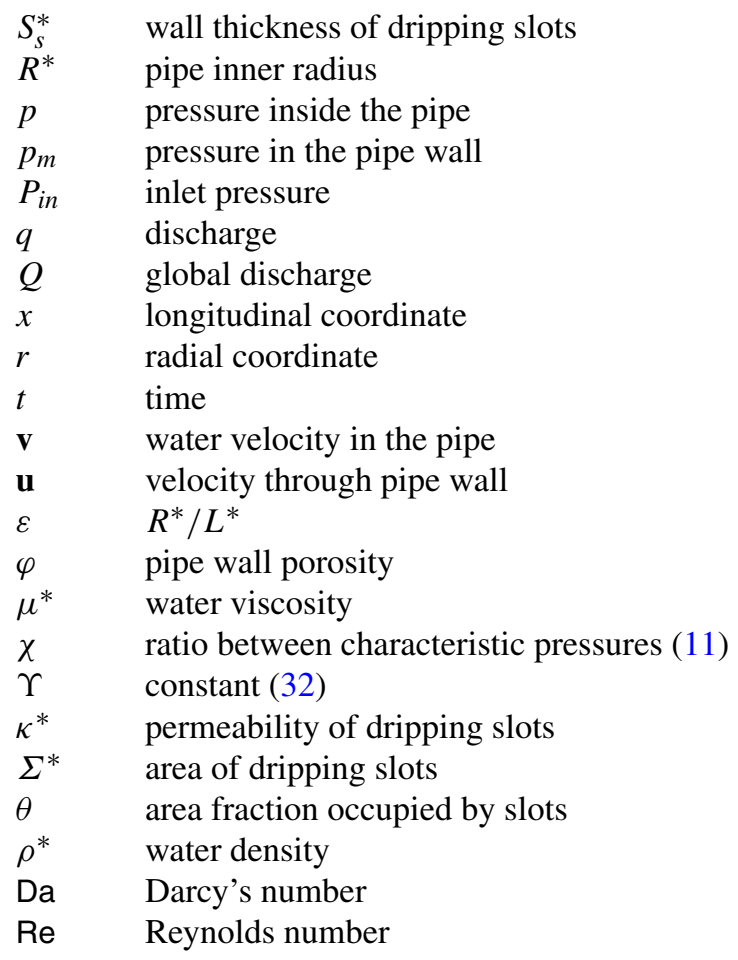

\section{Subscripts}

$\begin{array}{ll}r e s & \text { reservoir } \\ d & \text { dripping tubes } \\ c & \text { characteristic quantity }\end{array}$

\section{Introduction}

A widely used irrigation technique consists in delivering water by letting it filtrate through pipes laid down or suspended over the ground. Two types of plants are used, according to the size of the field to be irrigated. In small plants pipes made of a permeable and flexible material (e.g. canvas) and having length of the order of $100 \mathrm{~m}$ and radius of the order of $1 \mathrm{~cm}$ are laid on the ground and connected to a reservoir (a simple barrel) whose capacity is of the order of $1 \mathrm{~m}^{3}$ located in an elevated position (see Figure 1, right panel) so that the driving pressure is provided by gravity. In large plants pipes length is of the order of $1 \mathrm{~km}$, with a diameter of the order of $10 \mathrm{~cm}$. A much larger pressure is required, supplied by pumps, and therefore the pipe wall has to be thick and impermeable. Some artificial permeability is produced, for instance, by drilling dripping holes along the pipe (Figure 1, left panel), or by creating periodic permeable windows. In all cases the end of the pipe is sealed so that the flow takes place in the so called dead end configuration.

In the paper [1] we have studied in detail the dead-end flow in hollow fibers filtration modules. That study was promoted by the European Life + Project PURIFAST, 


\section{Irrigation pipes}

Large plants

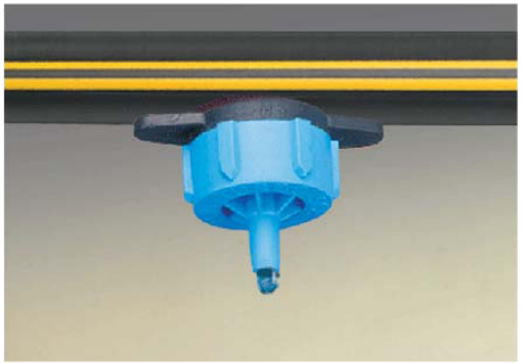

dripping pipes (thick)

Large pressure
Small plants

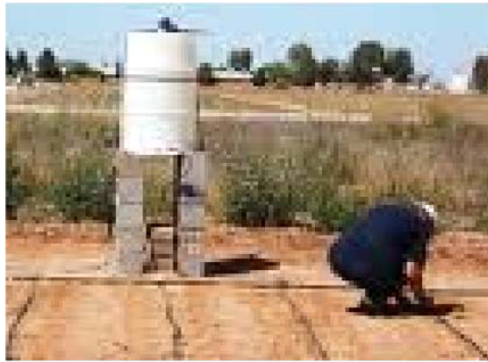

"exudating pipes" (thin)

Small pressure

Fig. 1 On the left: an example of hollow pipe used in large plants. On the right: a small plant.

and opened the way to a number of further investigations in different areas. Having acquired tools that are very appropriate for the study of flows through porous ducts, it seemed very natural to use them for the analysis of irrigation pipes.

The key point in designing an irrigation plant of both kinds is not only to regulate the total discharge, but also to obtain, at the far end of the pipe, a water delivery rate similar to the one close to the inlet. To this end the permeability (real or equivalent) of the pipe must be selected properly. As we shall see, more constraints may have to be imposed, if for instance the flow in the pipe is required to be laminar.

The technique we will use to obtain a reliable mathematical model of an irrigation pipe, inspired by [1], is the one of upscaling, that requires the following steps:

- write the Navier Stokes equation for the flow in the pipe, coupled with Darcy's law for the flow across the wall, together with the boundary conditions;

- exploit the smallness of the ratio $\varepsilon$ between the radius and the length of the pipe to expand all relevant quantities in power of $\varepsilon$;

- match the terms with equal powers in the differential system, and then average over the cross section to obtain a differential systems governing the macroscopic quantities at the various approximation orders.

In most cases the zero order approximation in $\varepsilon$ is quite satisfactory for practical purposes.

Clearly, here we are dealing with a low technology engineering problem that, to our knowledge, has never received attention from mathematical community. Therefore the related literature can be found in engineering journals and is addressed to the derivation of formulas of practical use, generally based on reasonable heuristic assumptions.

A pioneer paper on the subject was [2], describing the use of canvas hoses to deliver water to the ground, reducing evaporation and preventing soil erosion. More recent technical papers are $[3,4]$. However the idea of using porous materials in irrigation is very old (burying clay pots near the roots or, later, using clay pipes). Today 
the technique is used to such a large extent that periodic international conferences are held [5]. Buried porous pipes are also used for large scale applications [6].

The main difference between our approach and the quoted studies is that, in place of heuristic assumptions, we just apply the fundamental laws of dynamics of fluids in a pipe (Navier Stokes equations) and through porous media (Darcy's law). This is precisely the advantage of the upscaling procedure described above, which gives a sound rigorous basis to the theory.

In this paper we will analyze the low and high discharge pipes separately. The target we have in mind is to look for the physical and geometrical parameters which produce a prescribed discharge, while fulfilling additional constraints compatible with an effective operation of the plant.

\section{Low discharge pipes}

\subsection{The basic model}

The geometrical parameters can be chosen within some range. For the sake of being concrete, in our exposition we will use the following reference quantities: ${ }^{1} R^{*}=$ $5 \mathrm{~mm}$ (pipe internal radius), $L^{*}=100 \mathrm{~m}$ (pipe length), $S^{*}=1 \mathrm{~mm}$ (wall thickness). Therefore the ratio

$$
\varepsilon=\frac{R^{*}}{L^{*}}=5 \cdot 10^{-5}
$$

is well in the applicability range of an upscaling procedure.

The porosity and the permeability of the wall will be denoted by $\varphi$ and $K^{*}$, respectively. The spatial coordinates are: $x^{*} \in\left(0, L^{*}\right)$, and $r^{*} \in\left(0, H^{*}\right)$, where $H^{*}=R^{*}+S^{*}$, is the pipe external radius. In the dead end configuration the data to be prescribed are the inlet pressure $P_{i n}^{*}$ and the external pressure $P_{\text {ext }}^{*}$, that we set equal to zero. The water velocity $\mathbf{v}^{*}$ within the pipe is decomposed along the longitudinal and radial direction

$$
\mathbf{v}^{*}=v_{x}^{*} \mathbf{e}_{x}+v_{r}^{*} \mathbf{e}_{r},
$$

and the same is done for the velocity $\mathbf{u}^{*}$ through the porous wall

$$
\mathbf{u}^{*}=u_{x}^{*} \mathbf{e}_{x}+u_{r}^{*} \mathbf{e}_{r} .
$$

The other unknowns are the pressure $p^{*}$ in the pipe and $p_{m}^{*}$ in the pipe wall. For the moment we consider the time-dependent problem. The governing equations for $\left(\mathbf{v}^{*}, p^{*}\right)$ are:

(i) the incompressibility condition

$$
\nabla^{*} \cdot \mathbf{v}^{*}=0
$$

\footnotetext{
${ }^{1}$ Hereafter symbols with ${ }^{*}$ denote dimensional quantities.
} 
(ii) the Navier Stokes equation

$$
\frac{\partial \mathbf{v}^{*}}{\partial t^{*}}+\left(\mathbf{v}^{*} \cdot \nabla^{*}\right) \mathbf{v}^{*}=-\frac{1}{\rho^{*}} \nabla^{*} p^{*}+\frac{\mu^{*}}{\rho^{*}} \nabla^{* 2} \mathbf{v}^{*},
$$

where $\rho^{*}, \mu^{*}$ are the water density $\left(10^{3} \mathrm{Kg} / \mathrm{m}^{3}\right)$ and viscosity $\left(10^{-3} \mathrm{~Pa} \mathrm{~s}\right)$.

The equations for $\left(\mathbf{u}^{*}, p_{m}^{*}\right)$ are:

(iii) incompressibility

$$
\nabla^{*} \cdot \mathbf{u}^{*}=0
$$

(iv) Darcy's law

$$
\varphi \mathbf{u}^{*}=-\frac{K^{*}}{\mu^{*}} \nabla^{*} p_{m}^{*}
$$

Let us write the boundary conditions ${ }^{2}$

$$
\begin{aligned}
p^{*}\left(0, r^{*}, t^{*}\right) & =P_{i n}^{*}\left(t^{*}\right), \quad \text { (prescribed inlet pressure) } \\
p_{m}^{*}\left(x^{*}, H^{*}, t^{*}\right) & =0, \quad(\text { atmospheric pressure) } \\
p^{*}\left(x^{*}, R^{*}, t^{*}\right) & =p_{m}^{*}\left(x^{*}, R^{*}, t^{*}\right), \quad \text { (pressure continuity), } \\
v_{r}^{*}\left(x^{*}, R^{*}, t^{*}\right) & =\varphi u_{r}^{*}\left(x^{*}, R^{*}, t^{*}\right), \quad \text { (flux continuity) }, \\
v_{x}^{*}\left(x^{*}, R^{*}, t^{*}\right) & =0, \quad \text { (no-slip) }, \\
v_{x}^{*}\left(L^{*}, r^{*}, t^{*}\right) & =0, \quad \text { (dead-end) } \\
v_{r}^{*}\left(x^{*}, 0, t^{*}\right) & =0, \quad \text { (symmetry) } \\
\left.\frac{\partial v_{x}^{*}}{\partial r^{*}}\right|_{r^{*}=0} & =0, \quad \text { (symmetry). }
\end{aligned}
$$

We have already explained the meaning of conditions (1), (2). The interface condition (3) expresses the continuity of pressure, which is usual in such cases. Equation (4) is mass balance, expressed in the form of flux continuity, equation (6) is peculiar to the dead end configuration, equation (5) is the classical no-slip condition, and equations (7), (8) are consequence of the symmetry of the flow field around the pipe axis. In [1] interface conditions more general than no-slip have been examined, but (5) is adequate for the present case. Indeed an estimate of the Beavers-Joseph coefficient based on the specific values of porosity and permeability (see [1], for more details) shows that the slip effect plays no role at the zero order in $\varepsilon$.

\footnotetext{
${ }^{2}$ We are supposing that the terminal pipe section is made impervious. Nevertheless, this is a minor detail, since the relative contribution of any seepage from the terminal section to the total discharge is $\mathcal{O}(\varepsilon)$.
} 


\subsection{Dimensionless formulation}

A key point of the upscaling procedure is to introduce dimensionless variables by means of a double rescaling. Thus we define

$$
x=\frac{x^{*}}{L^{*}}, \quad r=\frac{r^{*}}{R^{*}},
$$

and set $H=H^{*} / R^{*}, S=S^{*} / R^{*}$. Then we introduce the characteristic longitudinal velocity $v_{c}^{*}$, according to the following criterion. We take a typical discharge of the plant $q_{c}^{*}$, and we define $v_{c}^{*}$ so that

$$
q_{c}^{*}=\pi R^{* 2} v_{c}^{*} .
$$

The rescaled velocity components are defined as follows

$$
v_{x}=\frac{v_{x}^{*}}{v_{c}^{*}}, \quad v_{r}=\frac{v_{r}^{*}}{\varepsilon v_{c}^{*}}, \quad u_{x}=\varepsilon \frac{u_{x}^{*}}{u_{c}^{*}}, \quad u_{r}=\frac{u_{r}^{*}}{u_{c}^{*}},
$$

where the characteristic velocity $u_{c}^{*}$ is taken so that $\varphi u_{c}^{*}=\varepsilon v_{c}^{*}$.

Next we define a characteristic time $t_{c}^{*}=L^{*} / v_{c}^{*}$, which we select as reference time scale. In particular, on the basis of (9) we have $t_{c}^{*}=\pi R^{* 2} L^{*} / q_{c}^{*}$, i.e. the ratio between the volume of the water filling the pipe and the characteristic discharge.

The inner channel pressure is rescaled by

$$
p_{c}^{*}=\frac{v_{c}^{*} \mu^{*} L^{*}}{4 R^{* 2}}
$$

obtained from considering a laminar flow of a Newtonian fluid, whose viscosity is $\mu^{*}$, in a tube of radius $R^{*}$ with the mean velocity $v_{c}^{*}$, where the factor $1 / 2$ has been introduced to simplify calculations in the sequel (we recall that the actual average pressure gradient in the dead end configuration is less than the one in the Poiseuille flow).

Concerning the membrane, we denote by $p_{m, c}^{*}$ the fluid characteristic pressure. We define it in the spirit of Darcy's law, i.e.

$$
p_{m, c}^{*}=\frac{\varphi \mu^{*}}{K^{*}} S^{*} u_{c}^{*}=\varepsilon \frac{\varphi \mu^{*}}{K^{*}} S^{*} v_{c}^{*} .
$$

We can express the ratio between the two characteristic pressures as

$$
\chi=\frac{p_{m, c}^{*}}{p_{c}^{*}}=\frac{\varepsilon^{2}}{8} \frac{S}{\mathrm{Da}}, \quad \text { with } \quad \mathrm{Da}=\frac{K^{*}}{R^{* 2}}, \quad \text { Darcy number. }
$$

Remark 1 From (11) we already have an indication about the value we can expect for Da. Since, for the reference data, $\varepsilon^{2} S=5 \cdot 10^{-10}$, if the two characteristic pressures have to be comparable, i.e. $\chi=O(1)$, we need $\mathrm{Da} \sim 10^{-9}$, indicating that $K^{*}$ of the order of $10^{-14} \mathrm{~m}^{2}$. 
The dimensionless version of the mathematical model illustrated above can be written as follows

$$
\begin{aligned}
& \frac{\partial v_{x}}{\partial x}+\frac{1}{r} \frac{\partial}{\partial r}\left(r v_{r}\right)=0, \\
& \frac{\operatorname{Re}}{\varepsilon}\left[\frac{\partial v_{x}}{\partial t}+v_{x} \frac{\partial v_{x}}{\partial x}+v_{r} \frac{\partial v_{x}}{\partial r}\right]=-\frac{4}{\varepsilon^{2}} \frac{\partial p}{\partial x}+\frac{\partial^{2} v_{x}}{\partial x^{2}}+\frac{1}{\varepsilon^{2}} \frac{1}{r} \frac{\partial}{\partial r}\left(r \frac{\partial v_{x}}{\partial r}\right), \\
& \frac{\operatorname{Re}}{\varepsilon}\left[\frac{\partial v_{r}}{\partial t}+v_{x} \frac{\partial v_{r}}{\partial x}+v_{r} \frac{\partial v_{r}}{\partial r}\right] \\
& =-\frac{4}{\varepsilon^{4}} \frac{\partial p}{\partial r}+\frac{\partial^{2} v_{r}}{\partial x^{2}}+\frac{1}{\varepsilon^{2}}\left(\frac{1}{r} \frac{\partial}{\partial r}\left(r \frac{\partial v_{r}}{\partial r}\right)-\frac{v_{r}}{r^{2}}\right), \\
& \frac{\partial u_{x}}{\partial x}+\frac{1}{r} \frac{\partial}{\partial r}\left(r u_{r}\right)=0, \\
& u_{r}=-\frac{S}{\chi} \frac{\partial p_{m}}{\partial r}, \\
& u_{x}=-\varepsilon^{2} \frac{S}{\chi} \frac{\partial p_{m}}{\partial x}, \quad v_{x=1}=0, \\
& \left.p\right|_{x=0}=P_{i n}(t)=\frac{P_{i n}^{*}(t)}{p_{c}^{*}},\left.\quad p\right|_{r=1}=\left.p_{m}\right|_{r=1},\left.\quad p_{m}\right|_{r=H}=0, \\
& \left.v_{r}\right|_{r=1}=\left.u_{r}\right|_{r=1},\left.\quad v_{x}\right|_{r=1}=0, \quad v_{x=0}=0, \\
& \left.v_{r}\right|_{r=0}=0,\left.\quad \frac{\partial v_{x}}{\partial r}\right|_{r=0}=0
\end{aligned}
$$

where

$$
\mathrm{Re}=\frac{\rho^{*} v_{c}^{*} R^{*}}{\mu^{*}},
$$

is the Reynolds number.

\subsection{Upscaling and zero order theory}

For each quantity in the system (12)-(20) we take the expansion

$$
f(x, r, t)=\sum_{n=0}^{\infty} f^{(n)}(x, r, t) \varepsilon^{n},
$$

and we match the terms with equal powers. In the dimensionless formulation the physical parameters enter just through the Reynolds number, whose order of magnitude is going to play an important role.

Looking at (13), (14) we realize that the inertia terms can be neglected at the zero order if the inequality $R e \ll \varepsilon^{-1}$, is satisfied. On the other hand, having used the Navier Stokes equations, we have implicitly assumed that the flow is laminar, thus 
$\operatorname{Re}<1000$. If $\varepsilon \sim 10^{-5}$, even $\operatorname{Re} \sim 1000$ allows us to ignore inertial terms in (13), (14). In practice, since the velocity decreases along the pipe, reducing to zero at the end, even $R e=2000$ can be acceptable.

Remark 2 Taking $\mathrm{Re}=1500$, the product $R^{*} v_{c}^{*}$ equals $1.5 \cdot 10^{-3} \mathrm{~m}^{2} / \mathrm{s}$ (recall that $\left.\mu^{*} \sim 10^{-3} \mathrm{~Pa} \mathrm{~s}\right)$. Thus for a radius $R^{*}=5 \mathrm{~mm}$ we have a total discharge $q_{c}^{*} \sim$ $9 \cdot 10^{-2} \mathrm{~m}^{3} / \mathrm{h}$, i.e. $2.2 \mathrm{~m}^{3} /$ day. Typical values in practice can be between 1 and $3 \mathrm{~m}^{3} /$ day. It corresponds to a time scale $t_{c}^{*}$ of approximately $3.3 \mathrm{~h}$.

From now on we argue as if the inertial terms were negligible in comparison with the terms having a factor $\varepsilon^{-k}$, with $k \geq 2$.

From (14) we immediately deduce that $p^{(0)}=p^{(0)}(x, t)$, (the same is true for $\left.p^{(1)}\right)$. Next, from (13) we derive the equation for $v_{x}^{(0)}$

$$
-4 \frac{\partial p^{(0)}}{\partial x}+\frac{1}{r} \frac{\partial}{\partial r}\left(r \frac{\partial v_{x}^{(0)}}{\partial r}\right)=0,
$$

whose solution (obtained by exploiting the boundary conditions $\left.v_{x}\right|_{r=1}=0$ and $\left.\left.\frac{\partial v_{x}}{\partial r}\right|_{r=0}=0\right)$ is

$$
v_{x}^{(0)}(x, r, t)=-\frac{\partial p^{(0)}}{\partial x}\left(1-r^{2}\right) .
$$

Note that the dead end condition implies $\left.\frac{\partial p^{(0)}}{\partial x}\right|_{x=1}=0$.

Now we go back to (12); this yields

$$
\frac{\partial}{\partial r}\left(r v_{r}^{(0)}\right)=\frac{\partial^{2} p^{(0)}}{\partial x^{2}} r\left(1-r^{2}\right),
$$

which, once integrated over $(0, r)$ gives

$$
v_{r}^{(0)}(x, r, t)=\frac{\partial^{2} p^{(0)}}{\partial x^{2}} \frac{r}{2}\left(1-\frac{r^{2}}{2}\right),
$$

which in turn, owing to $(19)_{1}$, delivers

$$
u_{r}(x, 1, t)=\frac{1}{4} \frac{\partial^{2} p^{(0)}}{\partial x^{2}} .
$$

Focusing next on (15)-(17), we get

$$
\frac{\partial}{\partial r}\left(r \frac{\partial p_{m}^{(0)}}{\partial r}\right)=0
$$

Hence, integrating over $r$ and using (18) 2 and (18) 3 we obtain

$$
p_{m}^{(0)}(x, r, t)=p^{(0)}(x, t)\left(1-\frac{\ln r}{\ln H}\right) .
$$


Now we recall (16)

$$
u_{r}^{(0)}=\frac{S}{\chi} \frac{1}{r \ln H} p^{(0)}
$$

and, by virtue of (24) and (25), the following equation for $p^{(0)}$ can be obtained

$$
\frac{\partial^{2} p^{(0)}}{\partial x^{2}}=4 \frac{S}{\chi} \frac{1}{\ln H} p^{(0)} .
$$

This is an ordinary differential equation with the boundary conditions $(18)_{1}$ and $\left.\frac{\partial p^{(0)}}{\partial x}\right|_{x=1}=0$ (dead-end). Since $H=1+S$, setting

$$
C^{2}=\frac{4 S}{\chi \ln (1+S)},
$$

we can express the pressure profile as follows

$$
p^{(0)}(x, t)=P_{\text {in }}(t)\left[\frac{e^{C x}}{1+e^{2 C}}+\frac{e^{-C x}}{1+e^{-2 C}}\right] .
$$

Hence, recalling (22) and (23), we have

$$
\begin{aligned}
& v_{x}^{(0)}(x, r, t)=P_{\text {in }}(t) C\left[\frac{e^{-C x}}{1+e^{-2 C}}-\frac{e^{C x}}{1+e^{2 C}}\right]\left(1-r^{2}\right), \\
& v_{r}^{(0)}(x, r, t)=\frac{P_{\text {in }}(t)}{2} C^{2}\left[\frac{e^{-C x}}{1+e^{-2 C}}+\frac{e^{C x}}{1+e^{2 C}}\right] r\left(1-\frac{r^{2}}{2}\right),
\end{aligned}
$$

respectively. Finally, from (25) we find

$$
u_{r}^{(0)}(x, r, t)=\frac{P_{\text {in }}(t)}{4 r} C^{2}\left[\frac{e^{-C x}}{1+e^{-2 C}}+\frac{e^{C x}}{1+e^{2 C}}\right],
$$

a decreasing function in $x$.

It is now easy to check that the global mass balance is verified at the zero order. Indeed, the dimensional discharge at the pipe inlet can be expressed as

$$
Q_{i n}^{*}=2 q_{c}^{*} \int_{0}^{1} r v_{x}(0, r, t) d r,
$$

and it must coincide with the lateral dimensional discharge, which is

$$
Q_{\text {out }}^{*}=2 q_{c}^{*} \int_{0}^{1} r u_{r}(x, 1, t) d x .
$$

With the help of (28) and of (30) it is easy to see that the two integrals coincide. In particular, we find

$$
\frac{Q_{\text {in }}^{*(0)}}{q_{c}^{*}}=\frac{Q_{\text {out }}^{*(0)}}{q_{c}^{*}}=\frac{1}{2}\left|\frac{\partial p^{(0)}(0, t)}{\partial x}\right|=\frac{1}{2} P_{\text {in }}(t) C \frac{\sinh (2 C)}{1+\cosh (2 C)} .
$$


Remark 3 It is interesting to compare $p^{(0)}(0, t)$ and $p^{(0)}(1, t)$

$$
\frac{p^{(0)}(0, t)}{p^{(0)}(1, t)}=\cosh C,
$$

which clarifies the physical meaning of $\cosh C$.

\subsection{Making the plant work}

Ground watering can be performed effectively only if the water delivery rate is distributed in a reasonably uniform way along the pipe. This requirement can be fulfilled by imposing that the ratio

$$
\Upsilon=\frac{u_{r}^{(0)}(1,1, t)}{u_{r}^{(0)}(0,1, t)}=\frac{1}{\cosh C}
$$

is sufficiently close to 1 . Thus the strategy in designing the pipe is to select first $\Upsilon$ (e.g. $\Upsilon=0.8$ ) and calculate $C$, according to (32). We get

$$
C=\ln \left[\frac{1}{\Upsilon}(1+\sqrt{1-\Upsilon})\right] .
$$

In turn, recalling (26), this is equivalent to selecting the ratio $\chi$, defined by (11), as

$$
\chi=\frac{4 S}{C^{2} \ln (1+S)} .
$$

We thus deduce the value of Da, namely

$$
\mathrm{Da}=\frac{\varepsilon^{2}}{32} C^{2} \ln (1+S)
$$

providing the wall permeability in terms of the geometrical data. For instance, if we take $\Upsilon=0.8$, we find $C \approx 0.7$, and finally obtain the wall permeability $K^{*}=$ $5 \cdot 10^{-15} \mathrm{~m}^{2}$, ensuring that at the end of the tube the water delivery rate is $80 \%$ of the one at the entrance.

Another piece of information that we can retrieve is the initial elevation of the water free surface in the reservoir, corresponding to a selected total discharge. To $Q_{\text {in }}^{*}=Q_{\text {out }}^{*}=2 \cdot 10^{-5} \mathrm{~m}^{3} / \mathrm{s} \sim 1.73 \mathrm{~m}^{3} /$ day, it corresponds $P_{\text {in }}^{*} \sim 18 \mathrm{KPa}$, i.e. an elevation of $1.8 \mathrm{~m}$.

This result, which actually agrees with the normal operating conditions (see Figure 1), is interesting, but it does not solve the whole problem yet. Indeed we still have to find how the inlet pressure $P_{i n}^{*}(t)$ evolves when the reservoir is progressively drained. ${ }^{3}$ This last step is very easy. Let the reservoir be a cylinder of radius $R_{\text {res }} r e s^{*}$

\footnotetext{
${ }^{3}$ Such a pressure can be maintained at its initial value by providing water at the same rate at which it is delivered to the ground along the whole pipe (which can be calculated since in that case the pressure is known). Alternatively, one can think of a periodic automatic (or manual) refill of the reservoir. Here we just consider a situation in which no extra water is supplied.
} 
and height $h_{r e s}^{*}$ and let be $h_{o}^{*}$ the elevation of its basis on the ground. The height $h^{*}\left(t^{*}\right)$ of the water level within the barrel will decrease in time and so will the pressure $P_{i n}^{*}\left(t^{*}\right)=\rho^{*} g^{*}\left(h^{*}\left(t^{*}\right)+h_{o}^{*}\right.$ ) (in the example above $h^{*}+h_{o}^{*}$ is about $2 \mathrm{~m}$, see Figure 1, right panel).

Equation (31) gives the total discharge in the form

$$
Q(t)=A P_{\text {in }}(t)
$$

where

$$
A=\frac{C}{2} \frac{\sinh (2 C)}{1+\cosh (2 C)},
$$

and

$$
P_{\text {in }}(t)=\frac{\rho^{*} g^{*} h_{r e s}^{*}}{p_{c}^{*}}\left(h(t)+h_{o}\right),
$$

having rescaled heights by $h_{r e s}^{*}$. The dimensional discharge must be equal to $-\pi R_{r e s}^{* 2} \frac{d h^{*}}{d t}$. Thus the following simple dimensionless equation is obtained

$$
\frac{d h}{d t}+A B\left(h+h_{o}\right)=0,
$$

where

$$
B=\frac{\pi R^{* 4} \rho^{*} g^{*}}{4 \mu^{*} q_{c}^{*}}\left(\frac{R^{*}}{R_{r e s}^{*}}\right)^{2} .
$$

If $R^{*} / R_{\text {res }}^{*}=10^{-2}$, and we keep the data used in the previous example, we find $B \sim 0.05$. The product $A B$ is of the order of $10^{-3}$, suggesting a dilation of $t$ by a factor $10^{3}$ (i.e. using hours instead of seconds for the dimensional time).

Thus the water level has an exponential decay and the time required to empty the reservoir can be easily calculated. Figure 2 shows the spatial profile of $P^{(0)}$ corresponding to different times and the exponential decay in time of water level in the reservoir.

\section{Large plants}

In large plants irrigation pipes are suspended over the ground and have lengths of the order of $1 \mathrm{Km}$. The possibility of extending the theory illustrated in the previous section to such long pipes relies on the following circumstance.

Remark 4 If the quantities $R^{*}, L^{*}, q_{c}^{*}$ are multiplied by the same factor, neither $\varepsilon$, nor the Reynolds number Re change.

Indeed, using (9) in (21) we find

$$
\mathrm{Re}=\frac{\rho^{*} q_{c}^{*}}{\pi \mu^{*} R^{*}} .
$$



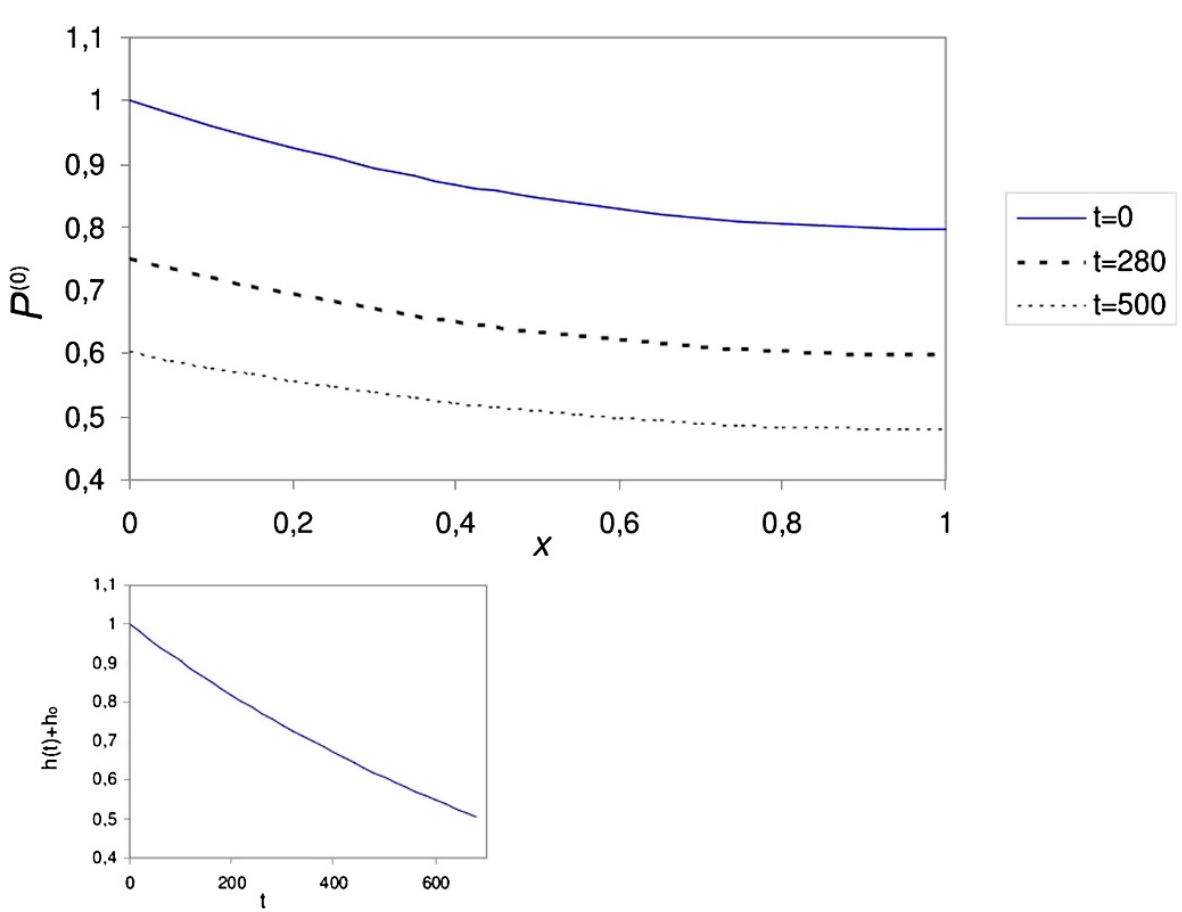

Fig. $2 P^{(0)}$ as function of $x$ when $C=0.7$. Time behavior of the water level $h(t)+h_{o}$, when $A B=10^{-3}$.

Therefore, passing from small to large plants may look like just a matter of scale. Multiplying the former characteristic quantities by a factor 10 we may consider the new reference values $R^{*}=5 \cdot 10^{-2} \mathrm{~m}, L^{*}=10^{3} \mathrm{~m}, q_{c}^{*}=2.510^{-4} \mathrm{~m}^{3} / \mathrm{s}$.

Nevertheless, there is not a minor difference, since a long pipe has to stand a much larger pressure, ${ }^{4}$ requiring thicker and impermeable walls. In this case permeability has to be produced artificially. There are various ways to do that. A technique we want to discuss here consists in drilling a periodic array of holes at the bottom of the pipe wall, each provided with a small tube from which water slowly drips to the ground. The flow in the small tube is approximately of Poiseuille type driven by pressure.

Since the inlet pressure is now constant, it make sense to consider just the steady state solution, dropping time dependence. Suppose that the holes are located at the coordinates $x^{*}=i \delta^{*}, i=1,2, \ldots, N$, with $N=L^{*} / \delta^{*}$, and that the dripping pipes have radius $R_{d}^{*}$ and length ${ }^{5} \ell_{d}^{*}$ (and we assume $\ell_{d}^{*}$ sufficiently larger than $R_{d}^{*}$, as in Figure 1, left panel). The water discharge of the $i^{\text {th }}$ hole is then ${ }^{6}$

\footnotetext{
${ }^{4}$ If the space scale ratio is 10 , then the typical value of $P_{i n}^{*}$ will be 2 bar.

${ }^{5}$ The length $\ell_{d}^{*}$ includes the pipe thickness $H^{*}-R^{*}$.

${ }^{6}$ For simplicity we take a constant flow. A more precise calculation should include the formation of a drop at the end of the tube, which grows until it falls down, like in the well known dripping faucet [7]. However, while surface tension provides a force that may give rise to the formation of drops in the gravity field, considering the order of magnitude of the driving pressure and the size selected for the dripping tubes, neither surface tension, nor gravity have any significant influence on the flow. Thus our approach is quite
} 


$$
q_{d, i}^{*}=\frac{R_{d}^{* 4}}{8 \mu^{*} \ell_{d}^{*}} p_{i}^{*}, \quad \text { where } p_{i}^{*}=p_{c}^{*} p^{(0)}\left(x_{i}\right), x_{i}=\frac{i}{N},
$$

with $p^{(0)}(x)$ given by (27). Here $p_{c}^{*}$ coincides to the entrance water pressure. We make the simplifying assumption that the flow in the pipe takes place as if the water loss is distributed all over the wall, to which an equivalent permeability is assigned. Such an assumption is justified by the fact that each tube carries a fraction of the order $1 / N$ of the total discharge, thus inducing a small perturbation of the flow regime considered in the previous section. Thus we apply the previous theory (supposing we are in the admissible range) in order to calculate $p_{i}$. In doing so we prescribe the total discharge $Q^{*}$ of the pipe. Then we impose that $Q^{*}=\sum_{i=1}^{N} q_{d, i}^{*}$. Passing to dimensionless variables, the latter equation takes the explicit form

$$
A=\mathcal{M} \sum_{i=1}^{N}\left(\frac{e^{C x_{i}}}{1+e^{2 C}}+\frac{e^{-C x_{i}}}{1+e^{-2 C}}\right)
$$

where $A$ is given by (33) and

$$
\mathcal{M}=\frac{R_{d}^{* 4}}{8 \mu^{*} \ell_{d}^{*}} \frac{p_{c}^{*}}{q_{c}^{*}}=\frac{1}{32 \pi}\left(\frac{R_{d}^{*}}{R^{*}}\right)^{4} \frac{L^{*}}{\ell_{d}^{*}} .
$$

By means of elementary techniques we can show that (with a very good approximation: recall that $N \gg 1$ )

$$
\sum_{i=1}^{N}\left(\frac{e^{C x_{i}}}{1+e^{2 C}}+\frac{e^{-C x_{i}}}{1+e^{-2 C}}\right) \approx \frac{N}{C} \frac{\sinh (2 C)}{1+\cosh (2 C)} .
$$

This formula can be obtained interpreting the 1.h.s. as a Riemann sum (since $N \gg 1$ ) and replacing it with the corresponding integral. Therefore, setting $\sigma_{L}=\ell_{d}^{*} / L^{*}, \sigma_{R}=$ $R_{d}^{*} / R^{*}$, we find the relationship

$$
\frac{\sigma_{L}}{\sigma_{R}^{4}}=\frac{N}{16 \pi C^{2}}
$$

In this equation $C$ is chosen according to the criterion exposed in the previous section (e.g. $C=0.7), N$ is chosen according to the desired frequency of the dripping holes (e.g. $2 / \mathrm{m}$ corresponds to $N=1000$, if $L^{*} \sim 0.5 \mathrm{Km}$ ). Therefore (36) says how to shape the dripping tubes (for instance, when $R^{*} \sim 2 \mathrm{~cm}, L^{*} \sim 0.5 \mathrm{Km}$, a consistent choice is $\ell_{d}^{*} \sim 5 \mathrm{~cm}, R_{d}^{*} \sim 1 \mathrm{~mm}$ ).

A very similar procedure allows to study the case in which the pipe wall has a periodic sequence of $N$ permeable slots of area $\Sigma^{*}$. The discharge through the slot

meaningful also for the following reasons: $(i)$ the dripping faucet problem is chaotic [8]; (ii) the dripping flows from the pipe are desynchronized; (iii) the ratio $q_{d, i}^{*} / Q^{*}$ is of the order of $1 / N \sim 10^{-3}$; therefore the local disturbance to the main flow is very small. 
located at position $x_{i}$ can be expressed as $q_{d, i}^{*}=\frac{\kappa^{*}}{\mu^{*}} \frac{p_{i}^{*}}{S_{s}^{*}} \Sigma^{*}$, where $\kappa^{*}$ is the permeability of the slots (the curvature of the wall and the longitudinal pressure drop can be neglected) to which we attribute the thickness $S_{s}^{*}$. Thus the expression for the total discharge contains once more the sum $\sum_{i=1}^{N} p_{i}^{*}$, which has the same approximated expression found before (35). Therefore, if we write the equation $Q^{*}=\sum_{i=1}^{N} q_{d, i}^{*}$, we find (as in previous case)

$$
q_{c}^{*} \frac{C}{2}=\frac{\kappa^{*} \Sigma^{*}}{\mu^{*} S_{s}^{*}} p_{c}^{*} \frac{N}{C} .
$$

Since, comparing (9) and (10), $\frac{q_{c}^{*}}{p_{c}^{*}}=4 \pi \frac{R^{* 4}}{\mu^{*} L^{*}}$, we find the following relationship

$$
\frac{\kappa^{*}}{R^{*} S_{S}^{*}}=C^{2} \frac{R^{* 3}}{32 N \Sigma^{*} L^{*}} .
$$

Introducing next the area fraction occupied by the slots, $\theta=\frac{N \Sigma^{*}}{2 \pi R^{*} L^{*}}$, we obtain $\frac{\kappa^{*}}{R^{*} S_{s}^{*}}=\frac{C^{2} \varepsilon^{2}}{64 \pi \theta}$.

For instance, if we take $C=0.7, \theta \sim 10^{-2}, \varepsilon=4 \cdot 10^{-5}$, the r.h.s. is $\sim 4 \cdot 10^{-10}$. With $R^{*} \sim 2 \mathrm{~cm}, S_{s}^{*} \sim 1 \mathrm{~mm}$, we eventually find $\kappa^{*} \sim 2 \cdot 10^{-14} \mathrm{~m}^{2}$. This corresponds to an apparent average permeability of the pipe of $\kappa^{*} \theta \sim 10^{-17} \mathrm{~m}^{2}$. We remark that what really matters is the ratio $\kappa^{*} / S_{s}^{*}$. Hence, the physical realization of the slots must be carried out so as to respect the desired value of $\kappa^{*} / S_{S}^{*}$.

\section{Conclusions}

We have adopted the so-called upscaling technique for obtaining the differential system governing the flow through pipes with porous walls used for irrigation purposes, in a dead-end configuration. The walls can be actually made of porous materials (e.g. canvas) when the pressure exerted on the pipe is of the order of $10^{4} \mathrm{~Pa}$ (small plants). In the case of large plants the walls are impermeable and an equivalent permeability is created, for instance, by drilling periodic holes from which dripping is controlled by selecting the hole radius and the length of dripping pipelets. Alternatively, dripping can be realized by means of a periodic distribution of porous slots.

The dynamics of both small and large plants is analyzed, deriving formulas of practical interest. For instance, one of the targets is to design the plant in such a way that the water delivery rate does not vary much from the entrance to the end of the pipe.

The advantage of the upscaling technique is that the derivation of the final equation proceeds rigorously from the basic laws of the fluid dynamics, so that the results obtained have a sound physical basis, rather than relying on heuristic assumptions. In our derivation we paid attention to practical applications, deriving formulas that can really be used in the design of an irrigation plant. 


\section{Competing interests}

The authors declare that they have no competing interests.

\section{Authors' contributions}

The authors have developed all the main sections of the paper jointly. Numerical calculations and related plots have been produced by A.F. All authors read and approved the final manuscript.

Acknowledgements This work was partially supported by the European Commission by means of the project PURIFAST (Advanced Purification of Industrial and Mixed Wastewater by Combined Membrane Filtration and Sonochemical Technologies, http://purifast.tecnotex.it/), within the program LIFE+ 2007 Contract N. ENV/IT/00439 - January 2009-December 2011.

\section{References}

1. Borsi, I., Farina, A., Fasano, A.: Incompressible laminar flow through hollow fibers: a general study by means of a two-scale approach. ZAMP 62, 681-706 (2011)

2. Robey, O.E.: Porous hose irrigation. Michigan Extension Bulletin 133, 1-22 (1934)

3. Zwieniecki, M.A., Thompson, M.V., Holbrook, N.M.: Understanding the hydraulics of porous pipes: tradeoffs between water uptake and root length utilization. J. Plant Growth Regul. 21, 315-323 (2003)

4. Mizushina, T., Takeshita, S., Unno, G.: Study of flow in a porous tube with radial mass flux. J. Chem. Eng. Jpn. 4, 135-140 (1971)

5. International Commission on Irrigation and Drainage: $7^{\text {th }}$ International Micro Irrigation Congress, Kuala Lampur, Malaysia, 2006

6. Lamm, F.R., Trooien, T.P.: Subsurface drip irrigation for corn production: a review of 10 years of research in Kansas. Irrig. Sci. 22, 195-200 (2003)

7. Dreyer, K., Hickey, F.R.: The route to chaos in a dripping water faucet. Am. J. Phys. 59, 619-627 (1991)

8. Rossler, O.: Nonlinear equations. In: Haken, H. (ed.) Synergistics: A Workshop, pp. 174-183. Springer, Berlin (1977) 